\title{
Fatores de Governança em Sistemas-de-Sistemas: Análise de uma Instituição Pública Brasileira
}

\author{
Marcio Imamura ${ }^{1}$, Luiz Alexandre Costa $^{1}$, Bruno Pereira ${ }^{1}$, Francisco Ferreira ${ }^{1}$, \\ Awdren Fontão ${ }^{2}$, Rodrigo Santos ${ }^{1}$ \\ ${ }^{1}$ Programa de Pós-Graduação em Informática \\ Universidade Federal do Estado do Rio de Janeiro \\ ${ }^{2}$ Programa de Pós-graduação em Ciência da Computação \\ Universidade Federal do Mato Grosso do Sul \\ \{marcio.imamura, luiz.costa\}@edu.unirio.br, \\ \{bruno.cesar, francisco.ferreira, rps\}auniriotec.br, \\ awdrendfacom.ufms.br
}

\begin{abstract}
Systems-of-systems (SoS) are sets of independent systems that collaborate and achieve an objective that cannot be achieved in isolation. SoS can be observed in several domains such as military, logistics, transportation, healthcare etc. Despite the great interest in this field in the last years, many problems are still open. Since the systems that compose an SoS are independent and have individual objectives, one of the most critical challenges is enabling the governance of SoS. Therefore, this paper presents an investigation of the factors that should be taken into consideration when implementing SoS governance. To do so, we conduct a survey with professionals of a Brazilian public organization.
\end{abstract}

Resumo. Sistemas-de-sistemas (SoS) são conjuntos de sistemas independentes que colaboram entre si para atingir um objetivo que não pode ser alcançado isoladamente. SoS podem ser observados em diversos domínios como: militar, logística, transporte, saúde etc. Uma vez que os sistemas que compõem um SoS são independentes e possuem objetivos próprios, um dos desafios mais críticos é viabilizar a governança para esse tipo de sistemas. Dessa forma, esse trabalho apresenta uma investigação acerca de fatores que devem ser considerados na implementação da governança em SoS. Para isso, foi realizada uma pesquisa de opinião com profissionais de uma instituição pública brasileira.

\section{Introdução}

Sistemas de informação (SI) são quaisquer combinações organizadas de pessoas, hardware, software, redes de comunicação, dados, políticas e procedimentos que armazenam, restauram, transformam e disseminam informações em uma organização [O'Brien e Marakas 2013]. Os principais desafios identificados pela comunidade de SI levaram a questões de como conceituar, construir e avaliar a nova geração de SI para lidar com a crescente complexidade técnica e diversidade social da sociedade contemporânea [Boscarioli et al. 2017].

Na sociedade, as empresas se organizam cada vez mais em redes colaborativas, estabelecendo alianças para atender mais rapidamente às demandas que surgem e aproveitar 
oportunidades de negócio, ao invés de construir novos SI isoladamente à partir do zero. Nestas alianças, SI independentes trabalham colaborativamente por meio de links de interoperabilidade entre si para formar arranjos que fornecem capacidades que não podem ser oferecidas por um SI de forma isolada. Tais arranjos são conhecidos como Sistemas-deSistemas ou SoS (Systems-of-Systems) [Maier 1998]. Um exemplo de SoS são as cidades inteligentes, onde diferentes organizações e SI trabalham em conjunto com o objetivo de melhorar a qualidade de vida dos cidadãos e otimizar a aplicação dos recursos públicos.

As estruturas existentes para gestão de SI tradicionais alcançaram um grau elevado de maturidade. Ferramentas e modelos como ITIL, COBIT, CMMI, MPS e PMBOK contribuem para controlar os riscos e os fluxos de informações associados à condução dos processos de negócio nas organizações [Ramlaoui e Semma 2014]. SoS, no entanto, apresentam características distintas dos SI tradicionais, sendo que cada sistema constituinte (SC) possui seus próprios objetivos, formas de governança, stakeholders e ciclos de vida. O conceito de governança está relacionado a padrões e relacionamentos construídos de forma estruturada e à implementação de ações que realçam um alinhamento das atividades operacionais com as diretrizes e objetivos estratégicos da organização [Tonelli et al. 2017]. Segundo Georgescu et al. (2019), a governança de sistemas complexos é um novo campo de estudos e pode não estar madura o suficiente para resolver os problemas existentes. Nesse contexto, existem desafios específicos a serem enfrentados no que diz respeito às formas de governança em SoS.

Este trabalho analisa fatores de governança no contexto de um SoS em operação em uma instituição pública brasileira que atua na área de produção de informações para planejamento. A instituição conta com 27 escritórios estaduais que coordenam o trabalho de cerca de 580 agências e 7.000 colaboradores. Foi realizada uma pesquisa de opinião com gerentes e desenvolvedores da instituição com o objetivo de investigar como acontece a governança neste SoS, utilizando os pilares de um framework para governança em SoS proposto por Darabi et al. (2013) .

\section{Fundamentação Teórica}

SoS possuem cinco características essenciais que os distinguem de outros sistemas [Maier 1998]: (i) independência operacional, pois os SC existem de maneira independente do SoS, sendo úteis por si só; (ii) independência gerencial, pois os SC são gerenciados de acordo com os objetivos próprios; (iii) distribuição geográfica, pois os SC não operam necessariamente em um mesmo ambiente físico; (iv) desenvolvimento evolucionário, pois o ciclo de desenvolvimento do SoS e dos SC pode ocorrer de maneira independente e contínua; e (v) comportamento emergente, gerando novas funconalidades por meio da sinergia entre os SC.

SoS são também classificados quanto à coordenação e existência de uma autoridade [Dahmann e Baldwin 2008, Maier 1998]. Um SoS pode ser: (i) dirigido, onde há uma autoridade central com poder coercitivo para executar o sistema; (ii) reconhecido, onde há uma entidade central, reconhecida pelos pares, que viabiliza a comunicação entre as partes para que se descubram SC e serviços prestados; (iii) colaborativo, onde os SC colaboram voluntariamente com o SoS e existe uma entidade gestora, mas sem poder coercitivo para executar o sistema; e (iv) virtual, que opera sem nenhuma autoridade gestora, dependendo de mecanismos não determinísticos para mantê-lo. 
A existência de mecanismos de coordenação e de uma autoridade gestora estão diretamente relacionadas à forma de governança. A governança de sistemas pode ser definida como a atividade responsável por garantir que os processos de TI estejam alinhados com a estratégia do negócio e agreguem valor para a organização [Van Grembergen e De Haes 2010]. Isso é feito por meio de normas, políticas e diretrizes pensadas para melhorar o desempenho do negócio, estabelecendo uma direção e fiscalizando seu cumprimento. Neste sentido, são definidos processos a serem seguidos e atribuídos papéis e responsabilidades aos participantes [Symons 2005]. No caso de SoS, as características de independência entre os SC bem como as diferentes formas de coordenação podem levar a situações onde não haja uma autoridade capaz de coordenar as ações e atribuir as responsabilidades, o que pode dificultar que sejam aplicados mecanismos de governança trandicionais a SoS.

Segundo Feeney et al.(2010), os sistemas de informação e de comunicação modernos são cada vez mais compostos por agregações altamente dinâmicas de subsistemas adaptativos ou autonômicos. Isso cria desafios significativos para o gerenciamento e governança em sistemas em que o comportamento dos componentes deva se adaptar de maneira que só se torne aparente após a implantação do sistema. De acordo com Darabi et al.(2013), estruturas de gerenciamento aplicadas à governança de sistemas tradicionais não são tão eficazes no contexto de SoS e estudos que investigam governança neste cenário ainda são escassos. Para fornecer uma estrutura de governança eficaz para um SoS, é necessário entender os mecanismos de governança em sua totalidade, observando seus componentes fundamentais em várias dimensões.

As dimensões propostas pelos autores foram reunidas em um framework para governança em SoS [Darabi et al. 2012], que traz princípios organizados em cinco pilares: (i) Integração de Propósito trata do fato de que qualquer SoS é projetado para servir a um objetivo e utiliza técnicas de negociação como forma de estabelecer e sustentar o propósito do SoS; (ii) Regulação de Pertencimento diz que o órgão de governança deve utilizar ferramentas para regular a entrada e saída de SC do SoS; (iii) Dispositivo de Incentivo significa que o órgão de governança deve utilizar mecanismos de incentivo para influenciar a gestão de recursos compartilhados; (iv) Protocolo de Interações diz que é de responsabilidade do órgão de governança definir e atualizar os protocolos de interações entre SC do SoS; e (v) Disseminação de Princípios e Alteração da Percepção é um componente que não utiliza a autoridade e pode ser percebido como um mecanismo social e informal de governança.

Para facilitar a classificação dos achados neste estudo, foi utilizado o conceito de SI vistos como sistemas socio-técnicos [Trist 1981]. Neste conceito, SI são formados por sistemas técnicos (relativos aos componentes tecnológicos) e sistemas sociais (relativos ao comportamento humano no trabalho) trabalhando em conjunto e gerenciados de forma equilibrada dentro de uma organização. O objeto de estudo deste trabalho foi um SoS caracterizado em uma instituição pública brasileira. A definição de governança de sistemas para organizações públicas e privadas é a mesma, sendo a arte de combinar e harmonizar as atividades que a área de TI desenvolve com as necessidades e objetivos estratégicos de uma organização, tornando a TI mais ativa no negócio [Weill e Ross 2006]. Enquanto que as empresas públicas devem assegurar que se agregue valor às ações da administração pública em benefício da sociedade, as empresas privadas devem assegu- 
rar a confiança dos investidores, aumentando a preocupação com a implementação de mecanismos de proteção para garantir a eficiência de processos e gerar competitividade. Assim, é possível aplicar o mesmo framework de governanca em SoS proposto por Darabi et al. (2013) tanto em organizações privadas quanto em organizações públicas, apesar de diferentes estratégias de negócio.

\section{Pesquisa de Opinião}

O SoS caracterizado neste estudo é composto por dois grupos de SI: técnicos que cuidam da produção de informações demográficas e econômicas e administrativos que cuidam do controle de recursos humanos e financeiros para realização do trabalho. A missão principal deste SoS é fornecer dados estratégicos para apoiar a tomada de decisão e acompanhamento da produtividade por meio da integração de dados desses grupos de SC. Todos os SC são desenvolvidos ou adquiridos de forma isolada, sendo que sua construção e evolução dependem de diferentes responsáveis e orçamento próprio, refletindo demandas específicas dos respectivos setores. Os SC técnicos cobrem as áreas de informações sobre população, emprego, atividade econômica e agricultura, enquanto os SC administrativos cobrem áreas como recursos humanos, contratos, controle de frota e viagens a serviço. Podemos considerá-lo um SoS dirigido, pois todos os SC estão subordinados à mesma instituição, embora a coodenação nem sempre seja exercida de maneira coercitiva pela autoridade central e sim de maneira colaborativa por meio de gestão entre departamentos.

Este estudo buscou analisar como ocorre a governança deste SoS, utilizando como referência os pilares do framework proposto por Darabi et al.(2013). A metodologia utilizada neste trabalho foi definida a partir de diretrizes da pesquisa experimental com o objetivo de avançar na investigação de como acontecem os mecanismos de governança em SoS. Para isto, foram utilizados os seguintes métodos experimentais: pesquisa de opinião para coletar opiniões e informações de um grupo de indivíduos e procedimentos de codificação e análise que fazem parte da Grounded Theory (GT) ou "Teoria Fundamentada em Dados" para analisar as opiniões e informações coletadas. A pesquisa de opinião foi planejada com o objetivo de elucidar como acontecem os mecanismos de governança no SoS caracterizado, procurando descobrir tópicos emergentes e dificuldades enfrentadas nesse contexto. Foram seguidos os procedimentos indicados por Linaker et al. (2015) e Molléri et al. (2016) para condução deste tipo de pesquisa.

\subsection{Instrumentação}

Para elaborar as questões da pesquisa de opinião, foram utilizados os cinco pilares de governança em SoS propostos em Darabi et al. (2013) para apresentar conceitos de governança e propor questões abertas sobre como acontecem os mecanismos de governança no contexto do SoS da instituição. Após os ajustes e adequações realizados por meio de um estudo piloto com uma aluna de mestrado em Informática, a pesquisa foi enviada para a amostra de profissionais selecionados. As questões abertas da pesquisa de opinião utilizando os cinco pilares de governança foram:

QA1 Como acontece a Integração de Propósito para o cenário de SoS da sua empresa? Você vê dificuldades e/ou facilidades nesta área?

QA2 Como acontece a Regulação de Pertencimento para o cenário de SoS da sua empresa? Você vê dificuldades e/ou facilidades nesta área? 
QA3 Como acontece o Dispositivo de Incentivos para o cenário de SoS da sua empresa? Você vê dificuldades e/ou facilidades nesta área?

QA4 Como acontece o Protocolo de Interações para o cenário de SoS da sua empresa? Você vê dificuldades e/ou facilidades nesta área?

QA5 Como acontece a Disseminação de Princípios e Alteração da Percepção para o cenário de SoS da sua empresa? Você vê dificuldades e/ou facilidades nesta área?

QA6 Você podria identificar outros pilares de governança existentes no contexto de SoS?

QA7 Comentários e/ou sugestões.

\subsection{Execução}

A pesquisa de opinião foi realizada no período de 28 de outubro a 01 de novembro de 2019. O questionário foi enviado para vinte profissionais de TI entre desenvolvedores, líderes de projeto e gerentes, envolvidos com as áreas de gerenciamento, desenvolvimento e de manutenção de SI da instituição. Dos vinte profissionais selecionados, nove responderam à pesquisa, obtendo-se assim um percentual de respostas de $45 \%$ da amostra. Essa taxa de resposta é considerada positiva para estudos desse tipo (surveys on-line) de acordo com Nulty (2008).

\section{Resultados}

Em relação ao grau de escolaridade dos participantes, todos os respondentes tinham nível superior, sendo três com graduação $(33,3 \%)$, um com especialização $(11,11 \%)$, três com mestrado $(33,3 \%)$ e dois com doutorado $(22,2 \%)$. Quanto ao nível hierárquico ocupado atualmente pelo respondente dentro da instituição, a maioria dos respondentes é composta por coordenadores/gerentes, com três respondentes (33,3\%), e por Analistas de Sistemas Plenos, também com três respondentes $(33,3 \%)$. Os demais cargos foram igualmente distribuídos com um Analista de Sistemas Junior/Trainee (11,1\%), um Analista de Sistemas Sênior $(11,1 \%)$ e um Desenvolvedor $(11,1 \%)$. Em seguida, buscou-se identificar o tempo de experiência na área de TI de cada respondente. A maioria (sete respondentes) trabalha na área há mais de 10 anos, totalizando $77,8 \%$, e apenas dois respondentes $(22,2 \%)$ possuem experiência entre 1 e 3 anos.

Para realizar a análise qualitativa dos dados, foram utilizados procedimentos de codificação e análise da GT com o objetivo de compreender as experiências e os significados que os atores vivenciaram em determinado cenário [Strauss e Corbin 2008], em conjunto com a ferramenta de software Atlas.TI ${ }^{1}$, específica para apoiar este tipo de atividade. Esta análise foi realizada com base nas respostas obtidas e foram aplicadas as codificações aberta e axial. Na codificação aberta, os dados foram analisados detalhadamente para a criação de códigos relacionados a trechos específicos das respostas.

Após a etapa de codificação aberta, buscou-se identificar as categorias (ou famílias, de acordo com a nomenclatura utilizada pela ferramenta Atlas.TI) e criar as relações e inter-relações entre os códigos, formando um encadeamento lógico, em um grau mais elevado. Nesta etapa, foram criados os códigos relacionados aos trechos selecionados das respostas diretamente ligados ao estudo. Foram identificados inicialmente

\footnotetext{
${ }^{1}$ https://atlasti.com/
} 
158 códigos livres que puderam ser consolidados em 124 códigos. Após análise e refinamento desta lista, 54 códigos foram selecionados pela relação direta com a governança em SoS de acordo com a proposta de Darabi et al. (2013). Para o agrupamento dos códigos encontrados, foi utilizada a visão de sistemas socio-técnicos [Trist 1981] e, assim, foram criados os grupos (i) fatores técnicos, (ii) fatores organizacionais; e (iii) fatores comportamentais. A Figuras 1, 2 e 3 apresentam a síntese dos fatores técnicos, organizacionais e comportamentais relacionados à governança de SoS.

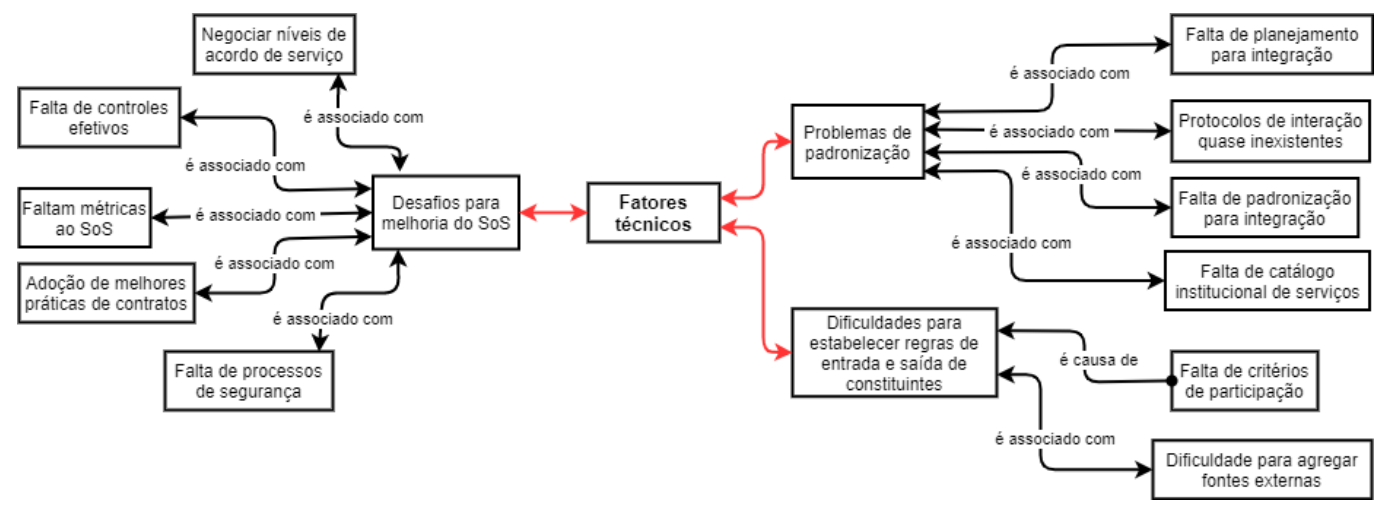

Figura 1. Fatores técnicos de governança em SoS

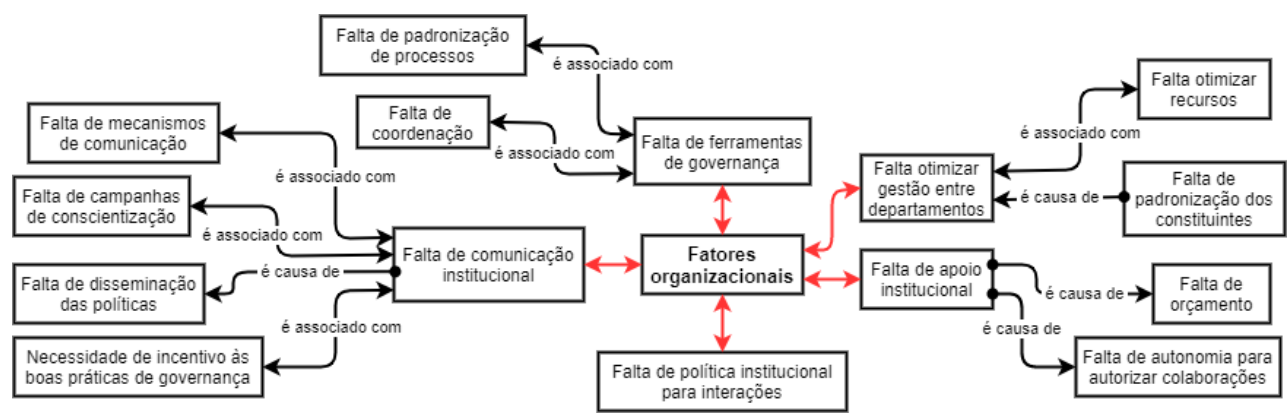

Figura 2. Fatores organizacionais de governança em SoS

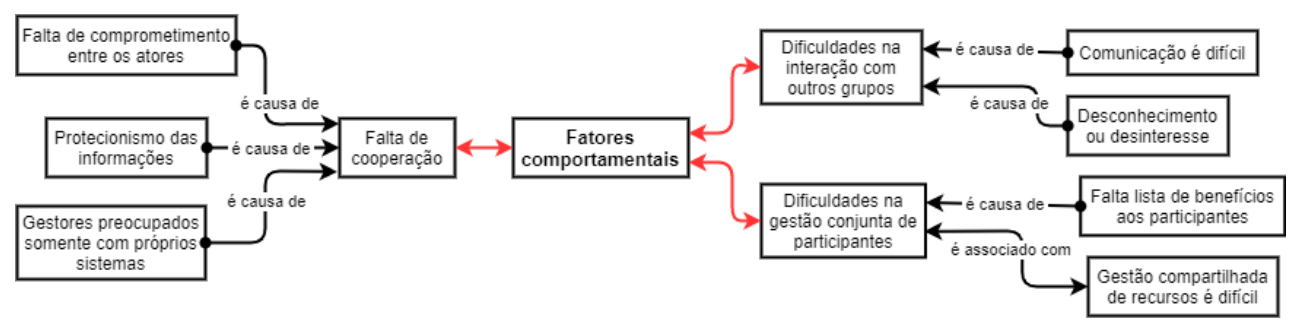

Figura 3. Fatores comportamentais de governança em SoS

\section{Discussão dos Resultados}

Percebe-se que as questões relativas à governança nas três categorias identificadas devem demandar maior ou menor atenção conforme o tipo de coordenação do SoS. Para SoS dirigidos, em que há uma autoridade central com poder coercitivo sobre os $\mathrm{SC}$, as decisões 
e mecanismos de governança devem ser facilitados, enquanto para SoS reconhecidos e colaborativos, a comunicação, o incentivo e o poder de persuasão são ferramentas extremamente importantes para garantir o bom funcionamento do sistema. Por sua vez, em SoS virtuais, não há como garantir nenhum mecanismo explícito de governança, pois não há sequer a garantia da comunicação entre os gestores ou operadores dos SC, impossibilitando tomar qualquer medida para garantir a continuidade e o bom funcionamento do SoS. Em cada uma das categorias identificadas, alguns fatores foram eleitos para aprofundamento da discussão, pois foram os mais citados pelos respondentes. Por exemplo, dentro da categoria de fatores técnicos, destacou-se a interoperabilidade, uma vez que esta questão precisa ser observada além dos critérios técnicos e tem sido pouco explorada, mesmo sendo relevante para a área de SI [Graciano Neto et al. 2017].

O grau de governança tem grande impacto na interoperabilidade, visto que é um elemento essencial quando se trata de SoS. Os SC de um SoS atingem um limite em ambientes colaborativos porque os métodos de gerenciamento de empresas são divergentes e estes sistemas possuem pacotes de recursos inflexíveis que não são criados com um objetivo de interoperabilidade. O desafio é torná-los interoperáveis para alcançar os ganhos necessários em competitividade e desempenho. Orientações para reusabilidade e redução das redundância de dados podem ser consideradas o ponto de partida para a interoperabilidade entre os SC. A falta de padronização em SoS foi discutida em [Vaneman e Jaskot 2013], onde os autores apontam um conjunto de regras, políticas e critérios de tomada de decisão que orientarão um SoS a atingir as suas metas e objetivos.

De acordo com os respondentes e os resultados gerados pela análise, percebem-se vários apontamentos na categoria de fatores organizacionais (i.e., falta de ferramentas de governança, falta de otimização de recursos e a falta de apoio institucional), que tornam evidente que a organização não está usufruindo dos benefícios de uma governança. A governança se consolida como um diferencial porque é capaz de expandir as possibilidades da empresa por meio da tecnologia, tornando-a mais competitiva [Webb et al. 2006]. Entre os benefícios, estão: (i) Maior precisão e segurança das informações, se a área de TI não consegue garantir que as informações estão seguras e são confiáveis, ela não consegue se sustentar por muito tempo; (ii) Melhor aproveitamento operacional e tecnológico por meio da definição de processos, sendo possível obter um melhor aproveitamento operacional e tecnológico e fazendo com que a TI invista tempo e recurso nas necessidades certas; e (iii) Otimização de custos sendo capaz de identificar as demandas prioritárias e trabalhar nas iniciativas corretas, gerando mais resultados ao negócio, reduzindo gastos com retrabalho e otimizando os custos.

Em relação à categoria de fatores comportamentais, um fator que merece atenção é a falta de cooperação entre os atores da organização (i.e., desenvolvedores, líderes de projeto e gestores). Segundo Maciel et al.(2008), pode emergir nas empresas uma limitação social para assegurar a cooperação dos indivíduos na consecução de um objetivo ou propósito. A cooperação é um dos principais elementos para o sucesso da organização e um dos fatores que contribuem para este objetivo é a construção de uma cultura organizacional. Um ambiente cooperativo gera benefícios diretos e indiretos para o negócio e não basta apenas reter talentos ou contar com profissionais qualificados. Contar com um ambiente participativo onde funcionários deem as mãos e unam forças é fundamental para o sucesso do SoS. Por isso, a cooperação é um fator determinante nesse contexto. 
Uma potencial alternativa de solução para a organização estudada é a implantação de um plano estratégico (i.e., ter envolvimento dos executivos da organização; atacar as principais vulnerabilidades a curto prazo; qualificar a equipe), cujo objetivo seria estabelecer qual o caminho mais adequado para a entrega de melhores resultados ao negócio [De Haes e Van Grembergen 2004]. No entanto, existem barreiras a serem superadas, destacando-se: a alta administração não compreende o valor agregado desta mudança; a alta administração e as áreas de negócio querem resultados práticos e em curto prazo; e as atividades do dia a dia não permitem implementar melhores práticas. Para a questão da cooperação organizacional, propõe-se melhorar a comunicação interna por ser crucial para as organizações de sucesso, pois afeta a capacidade dos gerentes estratégicos de envolver os funcionários e alcançar objetivos [Welch e Jackson 2007].

A proposta de elaborar um framework de governança em SoS realizada por Darabi et al. (2013) é inovadora e pode ser considerada uma das formas de agilizar o projeto de implantação de governança em uma organização, pois traz modelos prontos e testados na prática [Darabi et al. 2013]. Espera-se futuramente poder complementar essa proposta com os principais frameworks de processos para TI (i.e., ITIL $^{2}$ e COBIT $^{3}$ ) para que possa tornar o framework um padrão em governança de SoS.

\section{Limitações}

Apesar da taxa de resposta da pesquisa de opinião ser considerada positiva (45\% da amostra), entende-se que é importante obter mais respostas, tanto estudando meios de aumentar a amostra quanto deixando o questionário aberto por mais tempo, proporcionando um maior número de respondentes e visando maior contribuição ao cenário observado na organização.

Para trabalhos futuros, identificou-se a necessidade de conduzir entrevistas individuais com profissionais envolvidos com o contexto de SoS, aprofundando o entendimento das questões observadas com procedimento de codificação e análise da GT e procurando outras questões relativas à governança em SoS. Pode ser interessante conduzir um mapeamento sistemático da literatura para encontrar outras visões e ferramentas para governança em SoS capazes de enriquecer esta contribuição. Por fim, este estudo se limitou a analisar a governança em apenas um SoS de uma instituição pública. Analisar outros SoS, tanto em organizações privadas quanto em diferentes ramos de atividade, poderia contribuir para o melhor entendimento das questões de governança em SoS.

\section{Considerações Finais}

Este trabalho usou como base um estudo primário [Darabi et al. 2012] e buscou avaliar a percepção dos profissionais de TI de uma instituição pública sobre mecanismos de governança em SoS apontados como referência por meio de um framework. Para isso, foi realizada uma pesquisa de opinião com profissionais desta instituição que, em sua maioria, possuem nível de graduação ou mestrado, são coordenadores/gerentes e atuam há mais de 10 anos na área de TI. O estudo buscou identificar dificuldades, benefícios e fatores de influência de governança em SoS. Em seguida, foram aplicadas ferramentas

\footnotetext{
${ }^{2}$ Conjunto de boas práticas para governança de TI, com foco na gestão de serviços.

${ }^{3}$ Framework de gestão e governança de TI desenvolvido pela ISACA.
} 
para análise qualitativa das respostas. A análise realizada permitiu identificar três categorias principais: fatores técnicos, fatores organizacionais e fatores comportamentais. A partir da análise realizada, foi possível entender várias dificuldades relacionadas a cada um desses fatores.

Além disso, foi possível identificar desafios relacionados à melhoria dos SoS. Muitas carências foram apontadas como, por exemplo, a falta de processos de segurança, a falta de indicadores de desempenho, a falta de controles efetivos, a negociação de níveis de acordo de serviço, a falta de métricas e a adoção de melhores práticas de contratos. A análise qualitativa realizada contribui para o avanço na governança de uma determinada área de pesquisa, provendo indícios e hipóteses que podem ser testadas futuramente. Neste trabalho, a análise qualitativa contribuiu ainda para a identificação de tópicos deficientes do ponto de vista dos profissionais de TI da empresa.

Esta pesquisa de opinião considerou, como participantes, todos profissionais da área de TI que afirmam reconhecer a caracterização do SoS em questão. Contudo, o estudo apresenta algumas limitações: (i) não há garantia de que o entendimento das questões foi completamente absorvido pelos respondentes e ainda não se pode generalizar os resultados; (ii) a estrutura das perguntas da coleta de dados possuía poucas questões para as observações sobre a caracterização do cenário; e (iii) o processo de codificação dos dados qualitativos pode ter levado em consideração o viés dos pesquisadores que realizaram esta atividade; no entanto, para mitigar essa limitação, houve um rodízio na revisão dos resultados identificados entre os pesquisadores. Como trabalhos futuros, sugere-se: (i) investigar as relações entre os fatores identificados nos resultados; (ii) executar essa pesquisa em outra instituição para fortalecer os achados obtidos no presente estudo; e (iii) investigar a apresentação de benefícios individuais para stakeholders dos SC a fim de atraí-los ou convidá-los a participar de um SoS.

\section{Agradecimentos}

O presente trabalho foi realizado com apoio da Coordenação de Aperfeiçoamento de Pessoal de Nível Superior - Brasil (CAPES) - Código de Financiamento 001.

\section{Referências}

Boscarioli, C., Araujo, R. M., Maciel, R. S. P. (2017). I GranDSI-BR - Grand Research Challenges in Information Systems in Brazil 2016-2026. Special Committee on Information Systems (CE-SI). Brazilian Computer Society (SBC). 184p.

Dahmann, J. S. Baldwin, K. J. (2008). Understanding the current state of us defense systems of systems and the implications for systems engineering. In 2008 2nd Annual IEEE Systems Conference, pages 1-7. IEEE.

Darabi, H. R., Gorod, A., Mansouri, M. (2012). Governance mechanism pillars for systems of systems. In 2012 7th Int. Conference on System of Systems Engineering (SoSE), pages 374-379. IEEE.

Darabi, H. R., Mansouri, M., Gorod, A. (2013). Governance of enterprise transformation: case study of the faa nextgen project. In 2013 8th Int. Conference on System of Systems Engineering, pages 261-266. IEEE. 
De Haes, S. Van Grembergen, W. (2004). IT governance and its mechanisms. Information systems control journal, 1:27-33.

de Oliveira Maciel, C., Hocayen-da Silva, A. J., de Castro, M. (2008). Liderança e cooperação nas organizações. Revista de Economia e Administração, 7(3).

Feeney, K., Keeney, J., Brennan, R., O'Sullivan, D. (2010). An architecture for affective management of systems of adaptive systems. In IEEE Int. Workshop on Modelling Autonomic Communications Environments, pages 62-72. Springer.

Georgescu, A., Gheorghe, A. V., Piso, M.-I., Katina, P. F. (2019). Csi-a complex system governance approach. In Critical Space Infrastructures, pages 281-320. Springer.

Graciano Neto, V. V., Santos, R. P., Araujo, R. (2017). Sistemas de sistemas de informação e ecossistemas de software: Conceitos e aplicações. Tópicos em Sistemas de Informação: Minicursos SBSI, pages 22-41.

Maier, M. W. (1998). Architecting principles for systems-of-systems. Systems Engineering: The Journal of the Int. Council on Systems Engineering, 1(4):267-284.

Nulty, D. D. (2008). The adequacy of response rates to online and paper surveys: what can be done? Assessment \& evaluation in higher education, 33(3):301-314.

O’Brien, J. A. Marakas, G. M. (2013). Administração de sistemas de informação. AMGH.

Ramlaoui, S. Semma, A. (2014). Comparative study ocomparative of cobit with other it governance frameworks. Int. Journal of Computer Science Issues (IJCSI), 11(6):95.

Strauss, A. L. Corbin, J. (2008). Pesquisa qualitativa: técnicas e procedimentos para o desenvolvimento de teoria fundamentada. Artmed.

Symons, C. (2005). IT governance framework. Forrester Research.

Tonelli, A. O., de Souza Bermejo, P. H., Dos Santos, P. A., Zuppo, L., Zambalde, A. L. (2017). It governance in the public sector: a conceptual model. Information Systems Frontiers, 19(3):593-610.

Trist, E. (1981). The evolution of socio-technical systems. Occasional paper, 2(1981): 1981 .

Van Grembergen, W. De Haes, S. (2010). A research journey into enterprise governance of it, business/it alignment and value creation. Int. Journal of IT/Business Alignment and Governance (IJITBAG), 1(1):1-13.

Vaneman, W. K. Jaskot, R. D. (2013). A criteria-based framework for establishing system of systems governance. In 2013 IEEE Int. Systems Conference (SysCon), pages 491496. IEEE.

Webb, P., Pollard, C., Ridley, G. (2006). Attempting to define it governance: Wisdom or folly? In Proc. 2006 Annual Hawaii Int. Conf. System Sciences (HICSS'06), volume 8, pages 194a-194a.

Weill, P. Ross, J. W. (2006). Governança de TI: tecnologia da informação: como as empresas com melhor desempenho administram os direitos decisórios de TI na busca por resultados superiores. M. Books.

Welch, M. Jackson, P. R. (2007). Rethinking internal communication: a stakeholder approach. Corporate communications: An Int. journal, 12(2):177-198. 